\title{
CAPÍTULO 26: SECAGEM EM CAMADA DE ESPUMA E CARACTERIZAÇÃO FÍSICO QUÍMICA DA POLPA DE GUAVIRA (Campomanesia adamantium)
}

\section{CHAPTER 26: DRYING IN LAYER OF THE FOAM, AND THE CHARACTERIZATION OF PHYSICAL-CHEMICAL, PULP OF GUAVIRA (Campomanesia Adamantium)}

\author{
Djeiniffer Juliane Rodrigues ${ }^{1}$; Claudinéia Aparecida Queli Geraldi²; Raquel Aparecida Loss, ${ }^{3}$
}

\begin{abstract}
Resumo
A guavira (Campomanesia Adamantium) é uma fruta natural do cerrado, rica em vitamina $\mathrm{C}$, e disponível alguns meses do ano. Objetivou-se avaliar a cinética de secagem da polpa de guavira em camada de espuma, e a influência da temperatura em suas propriedades físico-químicas. A secagem foi realizada em três temperaturas 50,60 e $70^{\circ} \mathrm{C}$. Representando as curvas da cinética de secagem utilizou-se modelos matemáticos Henderson e Pabis, Midilli e Kucuk e Page. A polpa in natura e desidratada foram analisadas em relação ao teor de umidade, cinzas, vitamina C, acidez e pH. Destacou-se os modelos de Midilli e Kucuk e Page, com correlação superior a 0,99, o modelo de Page foi o que melhor representou os dados experimentais. O teor de umidade da polpa in natura foi de $81,37 \%$ e as polpas desidratadas a 50,60 e $70^{\circ} \mathrm{C}$ foram de $9,4 \%, 7,2 \%$, $4,0 \%$, atendendo à resolução RDC $\mathrm{n}^{\circ} 263$ da Anvisa. O teor de vitamina $\mathrm{C}$, cinzas e acidez aumentaram de acordo com a temperatura e o $\mathrm{pH}$ permaneceu inalterado. Recomenda-se a secagem a $70^{\circ} \mathrm{C}$, uma vez que o tempo de secagem é menor e a elevação da temperatura não comprometeu as características físico químicas da polpa.
\end{abstract}

Palavras-Chaves: Conservação; vida útil; sazonalidade.

\begin{abstract}
Guavira (Campomanesia Adamantium) is a fruit originally from Cerrado region, rich in vitamin $\mathrm{C}$ and only in season for some months of the year. This paper aimed at evaluating the kinetics of foam layer drying of Guariva pulp, and the influence of temperature on its physical-chemical properties. The drying process was carried out at three temperatures 50,60 and $70{ }^{\circ} \mathrm{C}$. In order to represent the drying kinetics curves, three mathematical models were used, Henderson and Pabis, Midilli and Kucuk, and Page. Both in natura and dehydrated pulps were analyzed for their moisture, ash, vitamin $\mathrm{C}$, acidity and $\mathrm{pH}$ contents. The Midilli and Kucuk and Page models stood out with a correlation greater than 0.99, the Page model was the one which best represented the experimental data. The moisture content of the in natura pulp was $81.37 \%$ whereas the moisture content of the dehydrated pulps at $50^{\circ} \mathrm{C}$ was $9.4 \%$, at $60^{\circ} \mathrm{C}$ was $7.2 \%$, and at $70{ }^{\circ} \mathrm{C}$ was $4.0 \%$, which is in accordance with the ANVISA resolution 263 . The contents of vitamin $\mathrm{C}$, ash and acidity increased according to the temperature, but the $\mathrm{pH}$ remained unchanged. It is recommended to dry at $70^{\circ} \mathrm{C}$, since the drying time is shorter and the rise in temperature did not compromise the physical-chemical characteristics of the pulp.
\end{abstract}

Keywords: Conservation; Self-life; seasonality.

\footnotetext{
${ }^{1}$ Engenharia de Produção Agroindustrial, UNEMAT: Universidade do Estado do Mato Grosso, djeini_juliane@hotmail.com

${ }^{2}$ Doutora, Faculdade de Arquitetura e Engenharia - Universidade do Estado de Mato Grosso, raquelloss@unemat.br

${ }^{3}$ Doutora, Faculdade de Arquitetura e Engenharia - Universidade do Estado de Mato Grosso, claudigeraldi@onda.om.br
} 


\section{Introdução}

Segundo a Secretaria de Agricultura e Abastecimento (SEAB), o Brasil ocupa a terceira colocação no ranking mundial da produção de frutas, com uma produção de 40,2 milhões de toneladas (SEAB, 2017). No entanto, os produtores enfrentam muitos problemas com relação a conservação destes alimentos devido ao excesso de umidade, oscilações na temperatura advindas das alterações climáticas com estações chuvosas ou secas, além de perdas ocasionadas pela ação do homem como, o transporte em grandes distâncias, manuseio incorreto ou excessivo e acondicionamento não adequado. Tais fatores favorecem a atuação de enzimas oxidantes resultando em alterações na composição das frutas (PEREIRA; BRAVIN; OLIVO, 2017). Outro fator que causa instabilidade para o setor é a sazonalidade do produto, representada principalmente em espécies de origem nativa, como a guavira, que amadurecem apenas nos meses de novembro e dezembro (EMBRAPA, 2015).

Campomanesia adamantium, comumente conhecido como "guavira" ou "guabiroba" floresce entre setembro e outubro, e frutificação entre novembro e dezembro. Seus frutos são saborosos, cítricos e levemente adocicados, sendo consumidos in natura ou usados na indústria alimentícia como aromatizantes na indústria de bebidas, licores, sucos, doces e sorvetes (OLIVEIRA et al., 2017).

A secagem é provavelmente um dos métodos mais antigo usado para preservar os alimentos. Frutas e vegetais são secos para inibir a atividade enzimática microbiana e a deterioração da qualidade. Como resultado da redução da atividade de água as mudanças físicas e químicas se minimizam durante o armazenamento aumentando o prazo de validade do material seco. Além de reduzir os custos de transporte e armazenamento uma vez que se reduz o peso e o volume do produto seco (ARAL e BESE, 2016). Diante da sazonalidade da guavira somado ao seu alto teor de água torna-se necessário desenvolver tratamentos pós-colheita, como a secagem, para preservar as suas características e disponibilizá-la para comercialização por períodos mais longos ou para posterior processamento.

A secagem em camada de espuma é um método relativamente simples e de baixo custo, que se baseia no uso de agentes estabilizadores de espuma durante a secagem. As vantagens deste método são as menores temperaturas e tempo de secagem, atribuídos à maior área de superfície exposta ao ar, permitindo maiores taxas de secagem em relação a outras técnicas de secagem (FRANCO et al., 2016).

Neste contexto o objetivo do presente estudo é aplicar a técnica de secagem em camada de espuma, verificando a influência da temperatura nas propriedades físico-químicas da polpa de guavira. Para alcançar o objetivo geral, os seguintes objetivos específicos foram delineados; 
a) obter a cinética de secagem da polpa de guavira em diferentes temperaturas. b) representar os dados de secagem por modelos matemáticos. c) determinar as características físico-químicas da polpa de guavira antes e após a secagem.

\section{Material e Métodos}

As análises foram realizadas nos laboratórios da Universidade do Estado de Mato Grosso, na cidade de Barra do Bugres - MT. Sendo eles, Laboratório de Química e Laboratório de Matérias Primas para Produção de Biodiesel (LMPPB).

Para o desenvolvimento deste trabalho utilizou-se como matéria-prima, a guavira, proveniente da cidade de Rio Brilhante, Estado de Mato Grosso do Sul - Brasil, e o aditivo emulsificante Emustab seleta (composto por monoglicerideos de ácidos graxos, monoestearato de sorbitana, polioxietileno de monoestearato de sorbitana e sorbato de potássio) adquirido no comércio local de Barra do Bugres - MT.

As frutas foram despolpadas e trituradas em um liquidificador doméstico até homogeneidade. Adicionou-se emulsificante na concentração de $4 \%$ em relação a massa de polpa e a mistura agitada em batedeira doméstica por 8 minutos em velocidade máxima, obtendo a espuma.

Na realização da secagem, a espuma foi distribuída em placas de Petri (10 g em cada placa) e submetida a estufa de circulação forçada de ar (Quimis 0314 M222), na qual algumas amostras foram acondicionadas a $50^{\circ} \mathrm{C}$, outras a $60^{\circ} \mathrm{C}$ e outras a $70^{\circ} \mathrm{C}$. Para a obtenção da curva de secagem, mediu-se a massa a cada 15 minutos até que a mesma ficasse constante. As curvas de secagem foram realizadas em triplicata. As massas obtidas foram convertidas em razão de umidade, conforme Equação 1.

$$
R U=\frac{U^{*}-U e^{*}}{U i^{*}-U e^{*}} \quad \text { Equação } 1
$$

Sendo, Ue* o teor de água de equilíbrio do produto; Ui* o teor de água inicial do produto; $\mathrm{U}^{*}$ o teor de água em um ponto qualquer da curva de secagem. Todas as umidades foram expressas em base seca.

Os dados da cinética de secagem, foram representados pelos modelos empíricos de Henderson e Pabis (1961), Page (1949) e Midilli e Kucuk (2002) (Tabela 1) para verificar qual se ajusta ao comportamento real da perda de umidade ao longo do tempo. A escolha do melhor 
modelo foi realizada por meio do coeficiente de determinação $\left(\mathrm{R}^{2}\right)$, pelo erro médio padrão e pelo princípio da parcimônia.

Tabela 1 - Modelos matemáticos utilizados para descrever a cinética de secagem.

DESIGNAÇÃO DO MODELO MODELO

\begin{tabular}{lc}
\hline Henderson e Pabis & $R U=a \exp (-k t)$ \\
\hline Page & $R U=\exp \left(-k t^{n}\right)$ \\
Midilli e Kucuk & $R U=\exp \left(-k t^{n}\right)+b t$ \\
\hline & Fonte: Elaborado pelos autores (2019).
\end{tabular}

Onde: RU razão do teor de umidade do produto, adimensional; t é o tempo de secagem, min; k, constante de secagem, $\min ^{-1}$, e a, b, n são os coeficientes dos modelos.

As amostras foram submetidas à análise de $\mathrm{pH}$, umidade, cinzas, acidez e vitamina $\mathrm{C}$ antes e após a secagem. Todas as análises foram realizadas em triplicata, para maior confiabilidade nos resultados obtidos.

Para a determinação do $\mathrm{pH}$ mediu-se $3 \mathrm{~g}$ de polpa de guavira diluída em 30mL de água destilada. O mesmo foi determinado por meio de um pHmetro de bancada (TECNOPON Mpa 210), previamente calibrado com solução tampão de pH 4,0 e 7,0, segundo o método 017/IV do manual do Instituto Adolfo Lutz (2008).

Na determinação de umidade mediu-se $10 \mathrm{~g}$ de amostra em placa de Petri, previamente tarada e seca, e colocadas em estufa com circulação de ar a $105^{\circ} \mathrm{C}$ até massa constante, conforme metodologia 012/IV do Manual do Instituto Adolfo Lutz (2008).

O teor de ácido ascórbico foi determinado a partir da metodologia 364/IV do manual do Instituto Adolfo Lutz (2008), na qual utilizou-se 5g de amostra diluída, 50mL de água destilada, com adição dos reagentes ácido sulfúrico a $20 \%$, iodeto de potássio a $10 \%$ e solução de amido a $1 \%$, e posteriormente titulou-se com iodato de potássio.

O teor de cinzas determinou-se conforme metodologia 018/IV do Instituto Adolfo Lutz (2008). Em cadinhos secos e tarados, foi medido $5 \mathrm{~g}$ de amostra e aquecidas na mufla a $550^{\circ} \mathrm{C}$, até obtenção de uma coloração branca ou acinzentada.

A acidez foi determinada por titulação potenciométrica com $\mathrm{NaOH} 0,1 \mathrm{~mol} / \mathrm{L}$, sendo os resultados expressos em \% de ácido cítrico, conforme método 016/IV do manual Instituto Adolfo Lutz (2008).

A comparação entre as médias foi realizada por meio de regressão linear e aplicação do teste de Tukey $(\mathrm{p}<0,05)$, utilizando softwares estatísticos. 


\section{Resultados e Discussão}

\section{Cinética de secagem}

As análises de curvas de secagem e a determinação do teor de água do produto permitem compreender melhor o processo, e deste modo possibilitam escolher o procedimento, tratamento, equipamento e temperatura adequados para se realizar a desidratação do alimento, com objetivo de manter a qualidade sensorial e tecnológica (SILVA, 2019). A Figura 1 representa os dados experimentais da cinética de secagem obtidas por meio secagem em estufa de circulação de ar, nas temperaturas as de 50,60 e $70^{\circ} \mathrm{C}$.

Figura 1 - Curva de secagem em camada de espuma da polpa de guavira em diferentes temperaturas

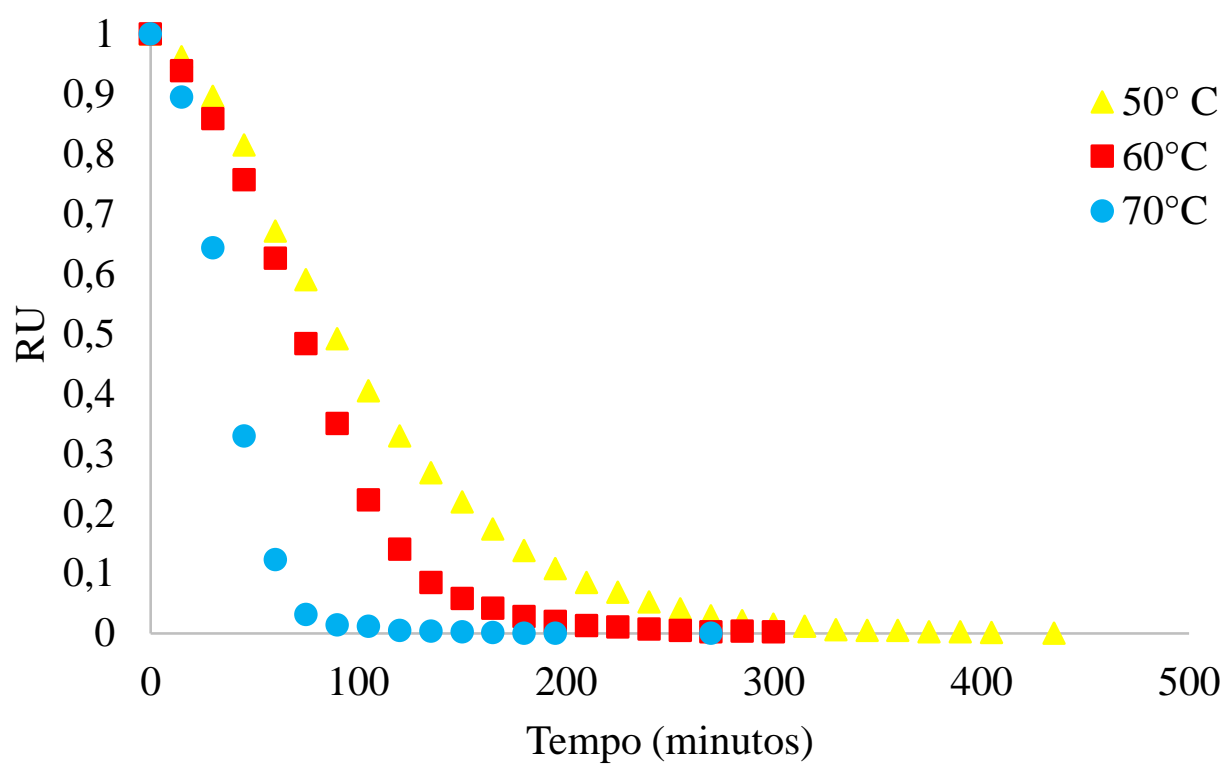

Fonte:

Elaborado pelos autores (2019).

As curvas da cinética obtidas apresentaram comportamento típico de secagem, conforme descrito por Fellows (2006), Silva (2019), e Celestino (2010). O início do processo é caracterizado pelo período de taxa constante, no qual água no alimento não apresenta nenhuma resistência para ser eliminada, uma vez que a água do interior do alimento difunde para a superfície deste na mesma velocidade da água evaporada, e este, permanece com a superfície úmida até a umidade crítica, representada na Tabela 2. 
Tabela 2 - Umidade crítica nas temperaturas 50,60 e $70^{\circ} \mathrm{C}$

\begin{tabular}{ccc}
\hline Temperatura & Tempo (min) & Umidade livre crítica $\left(\mathbf{K g H}_{2} \mathbf{O} / \mathbf{K g s s}\right)$ \\
\hline $\mathbf{5 0}^{\circ} \mathbf{C}$ & 300 & 1,051 \\
\hline $\mathbf{6 0}^{\circ} \mathbf{C}$ & 210 & 1,090 \\
\hline $\mathbf{7 0}^{\circ} \mathbf{C}$ & 105 & 1,118 \\
\hline
\end{tabular}

Fonte: Elaborado pelos autores (2019).

Após a umidade crítica, tem-se início o período de taxa decrescente, reduzindo o teor de umidade livre reduz além do crítico, 0,094, 0,073, 0,040 $\mathrm{KgH}_{2} \mathrm{O} / \mathrm{Kg}$ Ss, para as temperaturas de 50,60 e $70^{\circ} \mathrm{C}$, respectivamente, e a taxa de secagem se aproxima de zero, sendo chamado de ponto de equilíbrio, ou seja, o alimento fica em equilíbrio com o ar de secagem.

O tempo necessário para alcançar a umidade de equilíbrio foi maior para $50^{\circ} \mathrm{C}(300$ minutos), enquanto que esse tempo foi reduzido para 210 minutos para $60^{\circ} \mathrm{C}$ e 105 minutos para $70^{\circ} \mathrm{C}$. Esta diminuição no tempo de secagem pode ser explicada pela premissa de que o aumento da temperatura aumenta a pressão de vapor na amostra, e consequentemente causa a remoção da umidade do interior da polpa da guavira mais rapidamente à superfície. Aral e Bese (2016), encontraram a mesma diminuição no tempo de secagem após o aumento da temperatura em estudos da secagem de frutos do espinheiro branco.

As curvas de secagem foram representadas a partir de modelos matemáticos, sendo que, na Tabela 3 são apresentados os valores dos parâmetros estatísticos utilizados para a comparação entre os três modelos analisados por meio de regressão não linear. 
Tabela 3 - Parâmetros ajustados dos modelos para a cinética de secagem da polpa de guavira em camada de

\begin{tabular}{|c|c|c|c|c|}
\hline Modelo & Parâmetro & $\begin{array}{l}\text { espuma } \\
\mathbf{5 0}^{\circ} \mathbf{C}\end{array}$ & $60^{\circ} \mathrm{C}$ & $7^{\circ} \mathrm{C}$ \\
\hline \multirow{5}{*}{$\begin{array}{l}\text { Handerson } \\
\text { e Pabis }\end{array}$} & $\mathrm{a}$ & 1,274069 & 1,193782 & 1,000010 \\
\hline & $\mathrm{k}\left(\min ^{-1}\right)$ & 0,011723 & 0,016033 & 0,032443 \\
\hline & $\mathrm{n}$ & 0,000000 & 0,000000 & 0,000000 \\
\hline & $\begin{array}{l}\text { Coeficiente de } \\
\text { determinação R }\end{array}$ & 0,986068 & 0,973920 & 0,968662 \\
\hline & Erro & 0,982936 & 1,242921 & 0,820304 \\
\hline \multirow{5}{*}{$\begin{array}{c}\text { Midilike } \\
\text { Kucuk }\end{array}$} & $\mathrm{a}$ & 1,007082 & 0,999622 & 1,005116 \\
\hline & $\mathrm{k}\left(\min ^{-1}\right)$ & 0,000889 & 0,000289 & 0,000547 \\
\hline & $\mathrm{n}$ & 1,484823 & 1,821534 & 2,001636 \\
\hline & $\begin{array}{l}\text { Coeficiente de } \\
\text { determinação R }\end{array}$ & 0,999535 & 0,999319 & 0,999610 \\
\hline & Erro & 0,141440 & 0,209155 & 0,099886 \\
\hline \multirow{4}{*}{ Page } & $\mathrm{k}\left(\min ^{-1}\right)$ & 0,001022 & 0,000165 & 0,001509 \\
\hline & $\mathrm{n}$ & 1,457283 & 1,946679 & 1,733741 \\
\hline & $\begin{array}{c}\text { Coeficiente de } \\
\text { determinação R }\end{array}$ & 0,999498 & 0,999623 & 0,998097 \\
\hline & Erro & 0,142144 & 0,187894 & 0,215303 \\
\hline
\end{tabular}

Fonte: Elaborado pelos autores (2019).

Sendo: $\mathrm{k}$ o coeficiente de secagem $\left(\mathrm{min}^{-1}\right)$, a e $\mathrm{n}$ são constantes dos modelos e t: tempo de secagem (min).

Para verificar o grau de adequação dos modelos estudados aos dados experimentais obtidos na secagem em camada de espuma da guavira em diferentes temperaturas, observou-se a magnitude do coeficiente de determinação $\mathrm{R}$, os menores valores para o erro médio, bem como a quantidade de parâmetros de cada modelo.

Neste contexto é possível verificar que os modelos de Midilik e Kucuk, e Page, são os que possuem maior ajustes aos valores experimentais da secagem, isso por que nas três temperaturas obteve-se valores de R acima de 0,99. Além disso, segundo Cardoso et al. (2017) é importante ressaltar que para representar o fenômeno da secagem os valores aceitáveis de $\mathrm{R}$ devem ser superiores a 0,998. Desta forma, tanto o modelo de Midilik e Kucuk, quanto o de Page atende essas recomendações (Tabela 3). Resultados similares foram encontrados para a secagem de Moringa oleífera L. onde o modelo de Midilik e Kucuk, na temperatura $60^{\circ} \mathrm{C}$ foi a mais satisfatório (NASCIMENTO; BIAGI; OLIVEIRA, 2015). Moscon et al. (2017), observou evento semelhante na secagem de grãos de quinoa, nas temperaturas de 40,60 e $70^{\circ} \mathrm{C}$. Estudos 
realizados por Rigueto et al. (2018) analisando a cinética de secagem em camada de espuma da polpa de uvaia verificaram resultados semelhantes quanto à adequação dos modelos de Midilik e Kucuk e Page. Leal, Geraldi e Klassen (2016) ao analisarem a cinética de secagem em camada de espuma do abacaxi, acerola e carambola, nas mesmas condições de temperatura, também atestaram resultados semelhantes, como melhor adequação do modelo de Page.

$\mathrm{O}$ modelo de Handerson e Pabis foi o modelo que apresentou valores para R muito variados e disformes. No entanto, ao analisar a polpa de buriti Cardoso et al. (2017) constatou que este modelo foi mais representativo. Este fato pode ser explicado pelo fato de que a guavira como buriti são frutas de espécies diferentes, nativas e sazonais e, portanto, possuem comportamentos variados, além disso elas possuem composição de frutos diferentes.

Segundo Moscon et al. (2017), o coeficiente de determinação entre 0 e 1 mesmo mostrando a força de relação entre os dados, não constitui um critério suficiente se analisado de forma isolado. Deste modo avalia-se também o erro médio além dos parâmetros, a, k e n.

Os valores de erro médio relativo indicam desvio dos dados experimentais em relação à curva estimada pelo modelo, assim, o modelo que apresenta menor magnitude no que tange ao erro é o modelo com maior ajuste aos valores observados. O ideal é que o erro seja o mais próximo de zero, consideram-se modelos com valores de erro médio relativo superiores a $10 \%$ inadequados para a descrição de um determinado fenômeno de secagem, (ARAÚJO et al. 2017). Desta forma, em relação ao erro, todos os modelos utilizados podem ser considerados satisfatórios. No entanto, o modelo de Handerson e Pabis apresenta valores mais elevados. Enquanto que os modelos de Midilik e Kucuk e de Page apresentam erros próximos entre si, com exceção da temperatura de $70^{\circ} \mathrm{C}$, que o modelo de Midilik e Kucuk apresenta um erro menor. Resultados similares foram encontrados também na secagem em camada delgada de crambe a $45,60,75^{\circ} \mathrm{C}$ (COSTA et al., 2015), na secagem em camada de espuma de polpa de manga (GUIMARÃES et al., 2017) e também para secagem de folhas de hortelã (GASPARIN; CHRIST e COELHO, 2017).

Em se tratando dos parâmetros dos modelos tais como, "a", "k" e "n", pode se observar que o coeficiente "a" tende a diminuir conforme se aumenta a temperatura nos modelos Handerson e Pabis e Midiliki e Kucuk. Enquanto que, o coeficiente "k", possui tendência de ter seus valores aumentados conforme a alteração das temperaturas de 50 para $70^{\circ} \mathrm{C}$, isso ocorre devido a dependência deste parâmetro com a temperatura. Esse comportamento também foi observado por Alexandre (2005), em seu estudo de cinética de secagem em camada de espuma da polpa de pitanga, e por Silva et al. (2008), ao estudar cinética de secagem em camada de 
espuma da polpa de tamarindo e por Silva Filho et al. (2016) no estudo da cinética de secagem de polpa de manga.

No parâmetro "n” também é possível verificar dependência direta com a temperatura conforme aumento de 50 para $70^{\circ} \mathrm{C}$, este possui aumento considerável em seus valores. Esta dependência também foi constatada por Araújo et al. (2017) no estudo da secagem em camada de espuma da polpa de acerola ao testar o modelo de Page. A qualidade dos ajustes dos modelos aos dados experimentais pode ser melhor visualizada na Figura 2. 
RODRIGUES D.J., GERALDI C.A.Q e LOSS R.A.

Figura 2 - Modelagem dos dados experimentas pelos modelos de Handerson e Pabis, Midilick e Kucuk e Page em diferentes temperaturas: a) $50^{\circ} \mathrm{C}$; b) $60^{\circ} \mathrm{c} \mathrm{e} \mathrm{c)} 70^{\circ} \mathrm{C}$

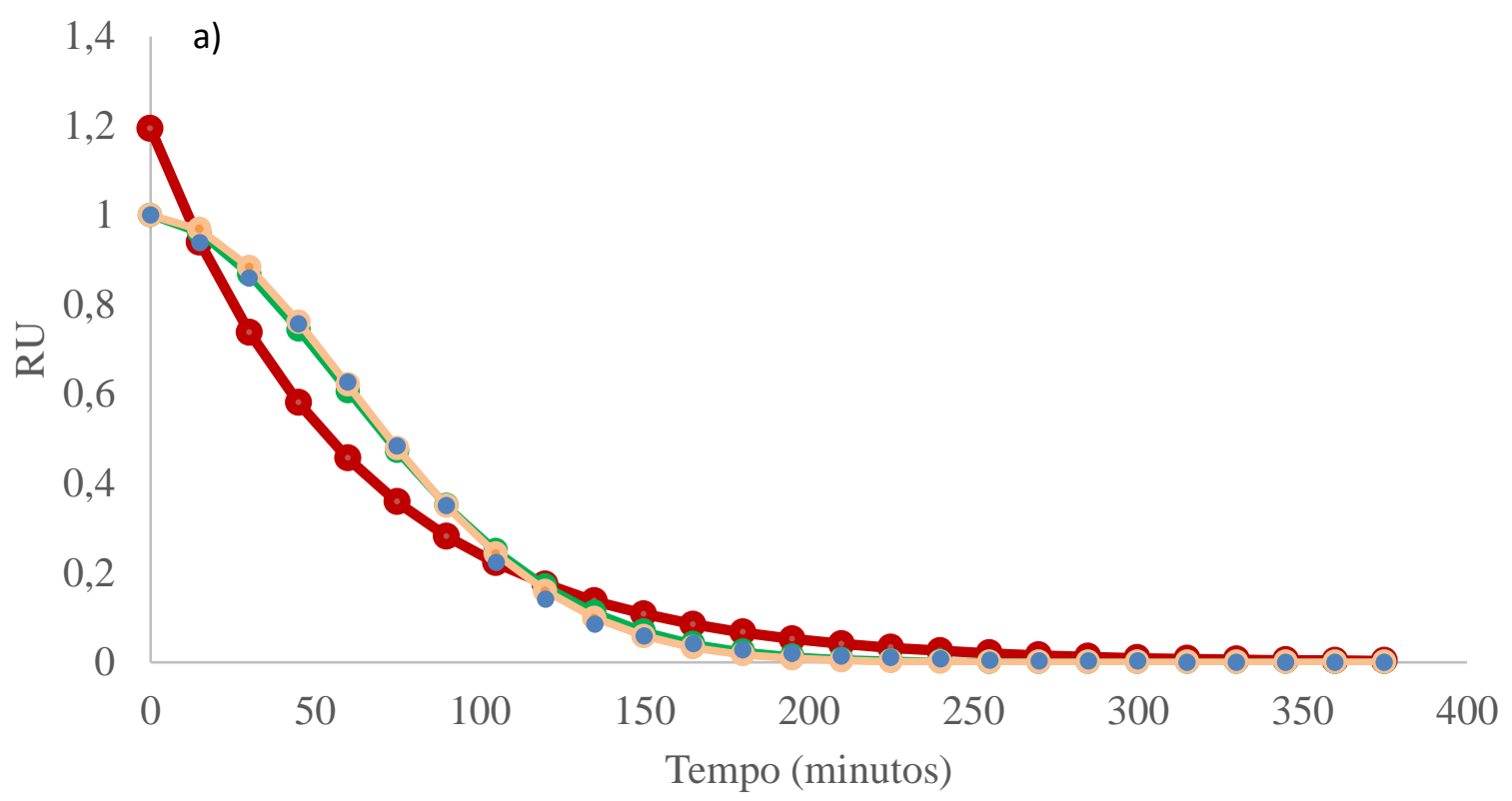

- Dados Experimentais -Handerson e Pabis —-Midilik e Kucuk —-Page

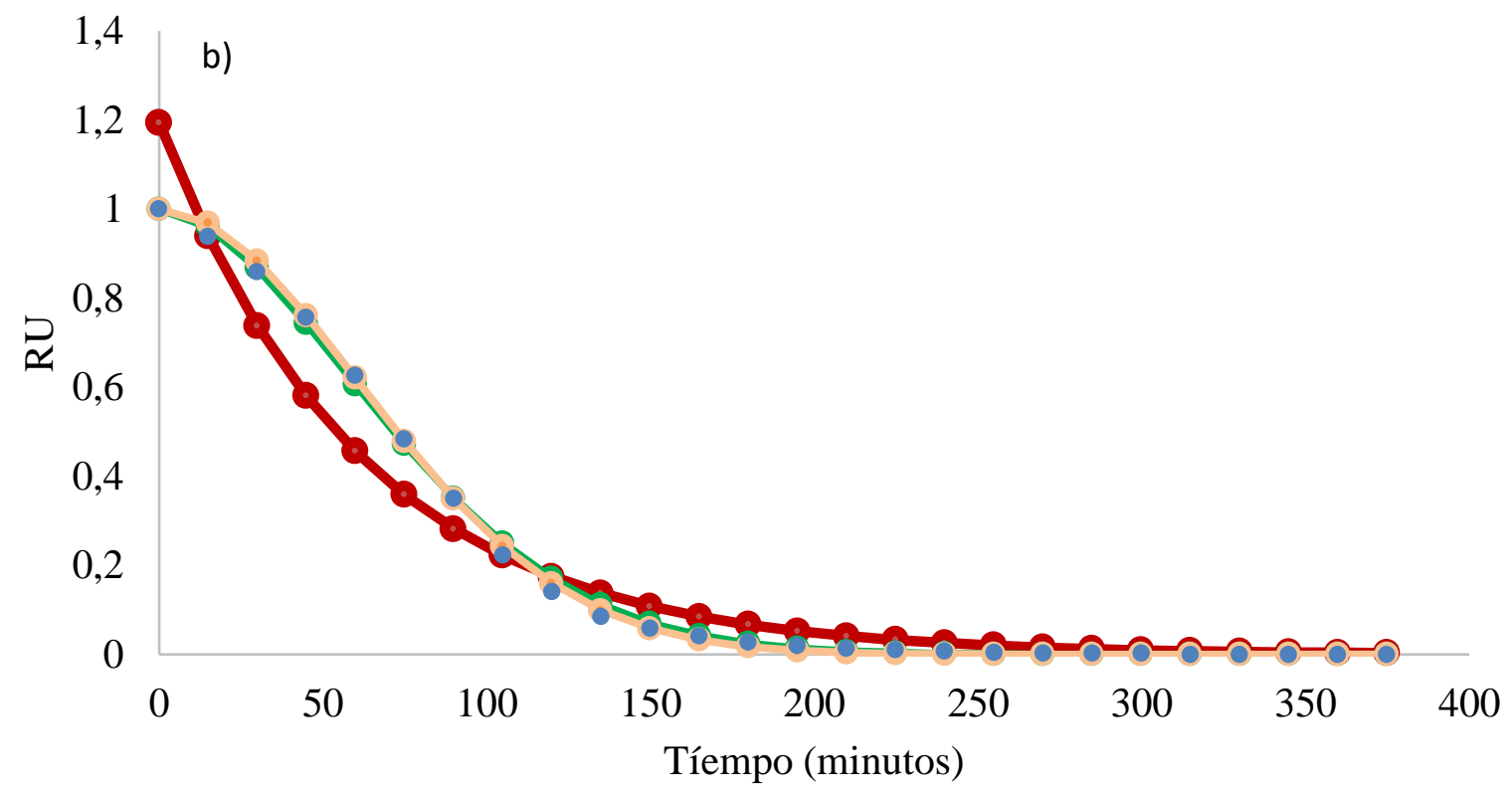

- Dados Experimentais - - Handerson e Pabis - - Midilik e Kucuk $-\odot-$ Page 


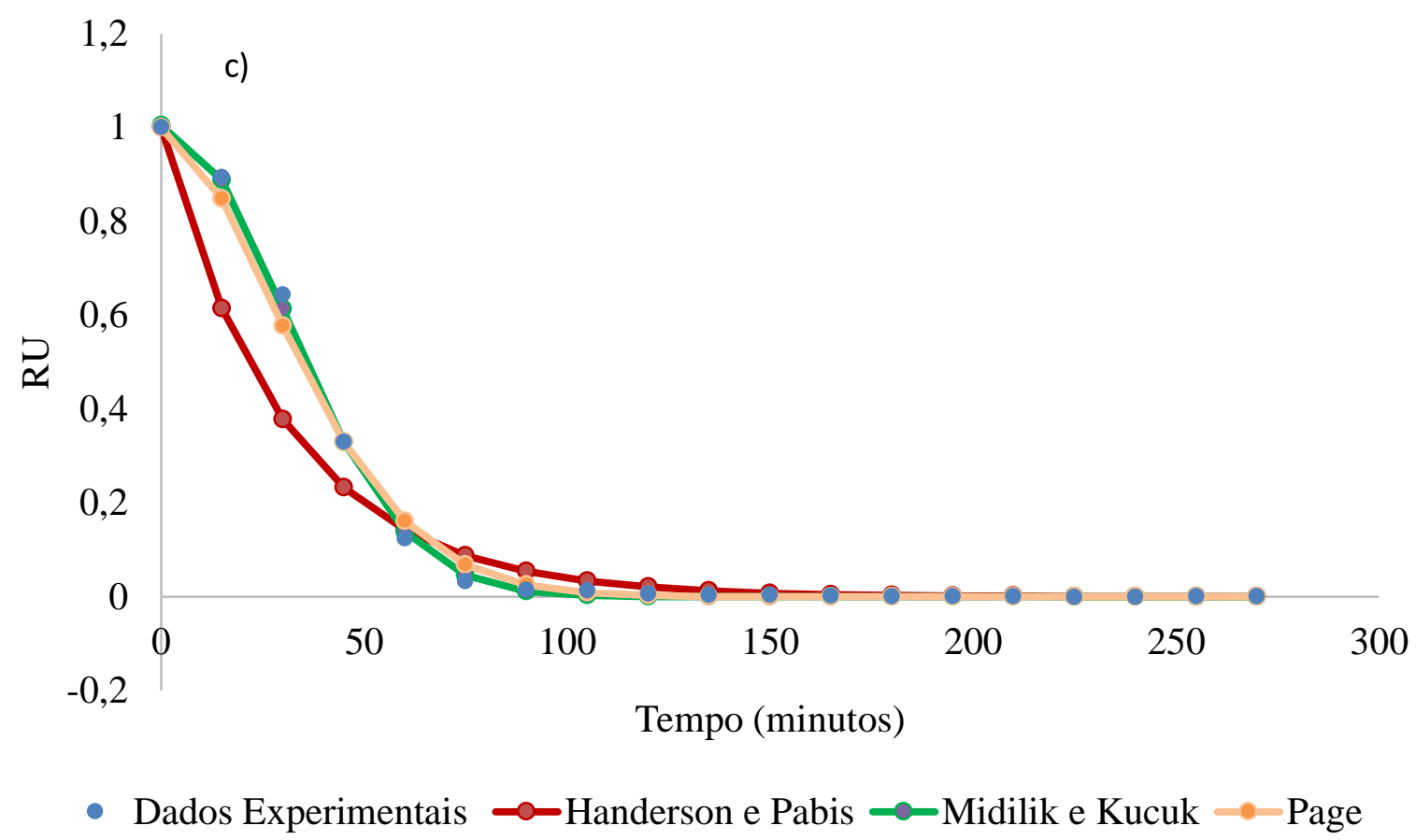

Fonte: Elaborado pelos autores (2019).

Por meio da Figura 2 é possível observar que tanto o modelo de Midilik e Kucuk quanto o modelo de Page se ajustam de forma satisfatória a curva dos dados experimentais. Contudo, no que tange ao número de parâmetros dependentes da temperatura o modelo de Page se sobressai em relação ao primeiro, uma vez que o mesmo possui apenas dois coeficientes, enquanto que o modelo de Midilik e Kucuk apresenta 4 coeficientes. Desta forma, o modelo de Page foi considerado o que melhor representa os dados experimentais.

\section{Análises Físico-químicas}

A guavira é uma planta nativa do cerrado brasileiro, presente nas regiões centro-oeste e sudeste, ela apresenta comportamento sazonal e pode ser consumida tanto na forma in natura, como na indústria de alimentos e bebidas. Seus frutos possuem atributos de qualidade como elevada acidez, ácido ascórbico (vitamina C), minerais, fibras alimentares e hidrocarbonetos, (VIEIRA et al., 2018). Desta forma as análises físico-químicas contribuem para o aprimoramento do conhecimento sobre a espécie, aproveitando suas formulações para agregar valor aos produtos desenvolvidos a partir dela (RESENDE et al., 2019). Neste contexto, os atributos analisados no presente estudo foram a vitamina $\mathrm{C}$, umidade, cinzas, acidez e $\mathrm{pH}$ na guavira in natura e desidratada. 
$\mathrm{O}$ pH é um indicador químico, que indica a concentração de íons de hidrogênio $\left(\mathrm{H}^{+} \mathrm{e}\right.$ $\mathrm{H}_{3} \mathrm{O}^{+}$) em um meio, sendo responsável pela característica ácida deste. Os valores inferiores a 7,0 indica meio ácido, valores superiores a 7,0 meio básico ou alcalino e igual a 7,0 meio neutro (CUNHA et al., 2016). Por meio da Tabela 4 é possível observar que o pH da amostra se manteve estável durante a desidratação, mostrando que a temperatura não influencia na alteração do $\mathrm{pH}$, isso ocorre devido a secagem ser um processo de retirada de água, que por sua vez, possui $\mathrm{pH}$ neutro, deste modo a retirada da mesma não altera o $\mathrm{pH}$ da amostra.

Tabela 4 - $\mathrm{pH}$ das polpas de guavira in natura e desidratadas em diferentes temperaturas

\begin{tabular}{ccc}
\hline Polpa & $\mathbf{p H}$ & $\mathbf{C V}(\boldsymbol{\%})$ \\
\hline in natura & $4,55 \pm 0,021^{\mathrm{a}}$ & 0,47 \\
$50^{\circ} \mathrm{C}$ & $4,51 \pm 0,040^{\mathrm{a}}$ & 0,93 \\
$60^{\circ} \mathrm{C}$ & $4,47 \pm 0,014^{\mathrm{a}}$ & 0,32 \\
$70^{\circ} \mathrm{C}$ & $4,47 \pm 0,014^{\mathrm{a}}$ & 0,32 \\
\hline
\end{tabular}

Letras iguais na mesma coluna, médias são iguais, pelo teste de Tukey ao nível de significância de $5 \%$ ( $<0,005)$. Fonte: Elaborado pelos autores (2019).

$\mathrm{O}$ pH da guavira entre 4,4 a 4,6 favorece a conservação da fruta, pois inibe a atividade de enzimas que causam oxidação e, na faixa de pH 4 e 4,5 a microbiota que se desenvolve no alimento é restrita, proporcionando maior tempo de prateleira para alimentos que apresentam este intervalo de pH (TELLES, 2012). Scalon et al. (2012) encontraram resultados similares ao analisar a polpa de guavira in natura, obtendo um $\mathrm{pH}$ variando entre 3,9 e 4,4, sob diferentes revestimento e temperaturas de armazenamento. Demenciano et al. (2015) ao analisar a polpa congelada da guavira registraram pH de 4,32 que é próximo ao encontrado neste estudo.

A umidade presente nas frutas é uma das características que a tornam altamente perecíveis pois, torna o ambiente favorável a proliferação de micro-organismo que oxidam e a putrificam. O alimento quando desidratado apresenta um percentual mínimo de umidade, denominada de umidade residual, que contribui para a inibição do crescimento de microorganismos patogênicos e deteriorantes. Na Tabela 5 pode-se verificar o teor de umidade encontrado na guavira in natura e os teores de umidade residual encontrado posterior a secagem.

Tabela 5 - Umidade da polpa de guavira in natura e desidratada em diferentes temperaturas

\begin{tabular}{ccc}
\hline Polpa & Umidade (\%) & CV (\%) \\
\hline in natura & $81,37 \pm 0,008^{\mathrm{a}}$ & 1.07 \\
$50^{\circ} \mathrm{C}$ & $9,40 \pm 0,001^{\mathrm{b}}$ & 1,08 \\
$60^{\circ} \mathrm{C}$ & $7,20 \pm 0,002^{\mathrm{c}}$ & 2,63 \\
$70^{\circ} \mathrm{C}$ & $4,00 \pm 0,002^{\mathrm{d}}$ & 4,69 \\
\hline
\end{tabular}

Letras iguais na mesma coluna, médias são iguais, pelo teste de Tukey ao nível de significância de 5\% (p<0,005).

Fonte: Elaborado pelos autores (2019). 
A umidade final obtida em todas as temperaturas, está de acordo com padrão de umidade estabelecido pela legislação brasileira, que determina um teor inferior a $25 \%$ em produtos de frutas secas ou desidratadas (ANVISA, 2005). Contudo a secagem do presente estudo foi realizada em manto de camada de espuma, ou seja, após a desidratação da polpa de guavira o produto obtido foi uma farinha. Neste contexto comparou-se os resultados obtidos no presente com a legislação estabelecida a produtos desidratados em forma de farinhas de origem vegetal, teor de $15 \%(\mathrm{~m} / \mathrm{m})$, constatando assim que o índice de umidade encontrado neste trabalho está de acordo com o preconizado pela ANVISA.

Poucos estudos com relação a caracterização físico-química de guavira (Campomanesia adamantium), nas mesmas condições são encontrados na literatura, porém comparando a cinética de secagem de frutas da mesma família, a família Myrtaceae, resultados similares são encontrados por Machado et al. (2012) ao estudar a cinética de secagem do abacaxi da variedade pérola, observou que a umidade residual a $70^{\circ} \mathrm{C}$ é bem menor comparado a demais temperaturas.

Santos (2018) afirma que leveduras e bactérias são susceptíveis a crescimento e manifestação em materiais com umidade em torno de $30 \%$. Portanto, o produto da desidratação de polpa de guavira é ideal para armazenamento, aumentando de forma considerável sua vida útil. Estes valores também foram encontrados com estudos de farinhas obtidas com sementes de araçá-amarelo e araçá-vermelho, através da secagem por meio de convecção natural, em temperatura ambiente, por Mayer (2015) que obteve índices de umidade de 7,56\% e 11,34\%.

O teor de cinzas trata-se da quantidade de substâncias minerais presentes nos alimentos, após a queima do material, retirando toda a matéria orgânica e umidade do produto restando somente o produto inorgânico. Na Tabela 6 estão apresentados os teores de cinzas obtidos por meio da calcinação da polpa de guavira, in natura e após desidratação em estufa de circulação de ar.

Tabela 6 - Teor de cinzas da polpa de guavira in natura e desidratadas em diferentes temperaturas

\begin{tabular}{ccc}
\hline Polpa & Cinzas $(\%)$ & CV (\%) \\
\hline in natura & $0,61 \pm 0,0001^{\mathrm{a}}$ & 1,41 \\
$50^{\circ} \mathrm{C}$ & $2,60 \pm 0,0003^{\mathrm{b}}$ & 1,42 \\
$60^{\circ} \mathrm{C}$ & $2,62 \pm 0,0002^{\mathrm{b}}$ & 0,84 \\
$70^{\circ} \mathrm{C}$ & $2,55 \pm 0,0003^{\mathrm{c}}$ & 1,56 \\
\hline
\end{tabular}

Letras iguais na mesma coluna, médias são iguais, pelo teste de Tukey ao nível de significância de 5\% (p<0,005).

Fonte: Elaborado pelos autores (2019). 
Pela Tabela 6 verifica-se que houve um aumento significativo no teor de cinzas do produto in natura para os desidratados, aumento de cerca de $226 \%$ de material inorgânico, ou seja, aumento de minerais na amostra. Segundo Wijewardana et al. (2016), este aumento no teor de cinzas ocorre devido a remoção de umidade que tende a aumentar a concentração de nutrientes. Elias et al. (2008) também encontrou aumento significativo no teor de cinzas após desidratação de polpa de caqui, aumento de cerca de 100\%, Nunes et al. (2017) ao analisar a cinética de secagem em estufa de circulação de ar forçada de resíduos de abacaxi obteve conteúdo de cinzas nos valores 2,82, 2,87 e 2,86 g (100g-1) nas temperaturas de 50, 60 e $70^{\circ} \mathrm{C}$ respectivamente, valores próximos aos encontrados neste estudo e nas mesmas condições de temperatura.

Outro fator que influenciou no conteúdo das cinzas foi a adição de emustab para a formação da espuma, para posterior secagem. O emustab que é um tipo de emulsificante tem em sua composição eletrólitos minerais, moléculas tensoativas, estabilizantes com cristais líquidos e macromoléculas dissolvidas na fase contínua, está composição implica no aumento da massa de material inorgânico da amostra, uma vez que ela auxilia na concentração de nutrientes (FERREIRA, 2011).

Rigueto et al. (2018), verificando a cinética de secagem da uvaia (Eugenia pyriformis) em camada de espuma, utilizando o mesmo método descrito no presente sob mesma condição de temperatura, também registrou a o aumento da concentração do conteúdo de cinzas da polpa in natura para a desidratada com teores de $0,24 \%$ para a amostra in natura e 2,62, 2,95, 3,24 $\%$ para os tratamentos de 50,60 e $70{ }^{\circ} \mathrm{C}$.

Um estudo publicado pelo IBGE - Instituto Brasileiro de Geografia e Estatística, com relação a composição dos alimentos (1999), apresentou teores centesimais (g/100g) de umidade e cinzas para a guavira, de $82,8 \%$ e $0,7 \%$, respectivamente, valores muito próximos a este estudo onde o conteúdo de umidade foi de $81,37 \%$ e cinzas $0,61 \%$.

A vitamina $\mathrm{C}$ é um micronutriente indispensável ao consumo humano, o Ministério da Saúde recomenda a ingestão diária de 45mg desta vitamina (BRASIL, 2004). Alguns estudos já haviam comprovado o elevado teor de ácido ascórbico presente nos frutos maduros de guavira assim como os valores encontrados no presente estudo e apresentados na Tabela 7. 
Tabela 7 - Teor vitamina $\mathrm{C}$ da polpa de guavira in natura e desidratadas em diferentes temperaturas

\begin{tabular}{ccc}
\hline Polpa & Vitamina C $(\mathbf{m g} / \mathbf{1 0 0 g})$ & CV $(\boldsymbol{\%})$ \\
\hline in natura & $629,29 \pm 15,5^{\mathrm{a}}$ & 2,46 \\
$50^{\circ} \mathrm{C}$ & $561,76 \pm 26,0^{\mathrm{b}}$ & 4,63 \\
$60^{\circ} \mathrm{C}$ & $894,42 \pm 44,4^{\mathrm{c}}$ & 4,97 \\
$70^{\circ} \mathrm{C}$ & $1438,48 \pm 3,2^{\mathrm{d}}$ & 0,22 \\
\hline
\end{tabular}

Letras iguais na mesma coluna, médias são iguais, pelo teste de Tukey ao nível de significância de 5\% (p<0,005).

Fonte: Elaborado pelos autores (2019).

O teor de vitamina $\mathrm{C}$ para a guavira in natura encontrado neste trabalho é alto em relação á valores encontrados na literatura, Dresch et al. (2015) e Vallilo et al. (2006) registraram um teor de $234 \mathrm{mg}$ em $100 \mathrm{~g}$ de polpa guavira. Este resultado está atribuído ao de fato de que no presente estudo a análise foi realizado com o conjunto polpa + casca, devido à dificuldade de extração da polpa. A casca por sua vez, assim como as folhas e frutos da guavira, também é rica em ácido ascórbico e demais composições. Segundo Oliveira et al. (2017) o conjunto folhas, casca, frutos de $C$. adamantium possuem propriedades medicinais, incluindo antiinflamatórias, antidiarréicas e anti-sépticas. Além disso, um estudo recente realizado por Alves et al. (2017) mostraram que frutos de guavira apresentaram maior teor de ácido ascórbico em comparação a outras espécies da família Myrtacea, como cagaita e cajueiro do cerrado.

Nota-se também na Tabela 8, que o conteúdo de ácido ascórbico diminui quando a polpa é desidratada a $50^{\circ} \mathrm{C}$, que pode ter ocorrido devido ao prolongado tempo de exposição da amostra nessa temperatura, um a vez que a exposição à luz e ao ar interferem negativamente na conservação do ácido ascórbico (CARDOSO et al., 2015).

Em oposto ao ocorrido na temperatura de $50^{\circ} \mathrm{C}$ nas temperaturas de 60 e $70^{\circ} \mathrm{C}$ este nutriente se concentrou apresentando um aumento significativo em comparação a amostra in natura. $\mathrm{O}$ aumento da vitamina $\mathrm{C}$ nestes tratamentos pode ser atribuído a crescente perda de massa ao longo das avaliações, como a amostra apresenta cerca de $80 \%$ de umidade, a perda da mesma contribuiu para a alta concentração do nutriente (OSHIRO, 2012). Comportamento similar foi observado por Leal, Geraldi e Klassen, (2016), ao analisar a secagem em camada de espuma do abacaxi, acerola e carambola, verificaram que o teor de vitamina tende a ser concentrado com o aumento da temperatura, inibindo as perdas por tempo de exposição.

O que caracteriza a guavira como uma fruta ácida além do $\mathrm{pH}$ abaixo de 7 , é a sua acidez, apresentada na Tabela 8. A acidez apresentou o mesmo comportamento que a vitamina $\mathrm{C}$, tendendo a se concentrar conforme aumentou-se a temperatura e diminuiu-se o tempo de exposição. Como já exposto a diminuição de massa de água faz com que nutrientes se concentrem, assim, quanto menor a umidade do produto maior sua concentração nutricional. 
Tabela 8 - Acidez da polpa de guavira in natura e desidratadas em diferentes temperaturas

\begin{tabular}{ccc}
\hline Polpa & Acidez $(\%)$ & CV (\%) \\
\hline in natura & $8,27 \pm 0,16^{\mathrm{a}}$ & 1,96 \\
$50^{\circ} \mathrm{C}$ & $18,66 \pm 0,15^{\mathrm{b}}$ & 0,59 \\
$60^{\circ} \mathrm{C}$ & $26,37 \pm 0,48^{\mathrm{c}}$ & 2,62 \\
$70^{\circ} \mathrm{C}$ & $29,09 \pm 0,31^{\mathrm{d}}$ & 1,08 \\
\hline
\end{tabular}

Letras iguais na mesma coluna, médias são iguais, pelo teste de Tukey ao nível de significância de 5\% (p<0,005).

Fonte: Elaborado pelos autores (2019).

Observa-se que, por mais que o $\mathrm{pH}$ permaneça quase inalterado nos três tratamentos propostas ainda assim o teor de acidez aumentou em relação a amostra in natura. Breda (2012), ao verificar a vida útil da guavira em pó desidratada pelo método de camada de espuma em ambientes controlados, registrou que, embora a acidez do produto em pó variasse de $15 \%$ a $25 \%$ o pH também permanecia na faixa de 4,12 a 4,7, apresentando variações somente após 80 dias de armazenamento. O que significa que apesar de, as concentrações de ácidos totais aumentarem em decorrência a desidratação, este aumento só implicara alterações no $\mathrm{pH}$ conforme algum tempo de armazenamento.

Muitos são os fatores que explicam a elevada acidez e constância do pH nas amostras, como por exemplo o método utilizado para determinação de acidez. Este método, se limita a valores de acidez detectáveis por titulação e as substâncias responsáveis por esta acidez detectável aparente são: os fosfatos e citratos (minerais), a caseína e albumina (proteínas) e gás carbônico dissolvido. Sabendo que a guavira é rica em sais e minerais alcalinos, que por sua vez tem ação neutralizadora de pH (BACCAR, 2016), é possível detectar acidez na polpa da fruta mesmo mantendo seu $\mathrm{pH}$ inalterado.

Outro componente detectável por meio de titulação é o ácido cítrico que é um ácido orgânico fraco, frequentemente encontrado nas frutas, sua acidez é devida aos três grupos carboxilas - $\mathrm{COOH}$ que podem perder um próton em soluções. Como consequência forma-se um íon de citrato, os citratos, por sua vez são controladores de pH nas soluções ácidas (FOOD INGREDIENTS BRASIL, 2014). Deste modo explica altas concentrações de acidez detectada na titulação, mesmo sem a alteração do $\mathrm{pH}$.

Um grande número de espécies da família Myrtaceae apresentam ocorrências de compostos fenólicos de estrutura simples, como os ácidos fenólicos ou taninos hidrolisáveis (ROCHA et al., 2011). Ácidos fenólicos são outro exemplo de como conteúdo de acidez pode ter sido concentrado na fruta conforme retirou-se a umidade. 


\section{Conclusões}

No que se refere a cinética de secagem observou-se que o tempo de secagem diminui de acordo com o aumento da temperatura. Quanto a adequabilidade dos modelos matemáticos constatou-se que os modelos de Midilli e Kucuck, e Page se sobressaem em relação ao de Handerson e Pabis, em todos os critérios verificados, o coeficiente de determinação. Contudo o modelo de Page foi escolhido para representar os dados experimentais pois possui menor número de parâmetros.

As características físico-químicas da farinha de polpa de guavira apresentaram comportamento similar estre elas, as quais após a secagem foram concentradas apresentando um aumento significativo no teor de cinzas, vitamina $\mathrm{C}$ e acidez em relação ao da polpa in natura. O teor de umidade após a obtenção da farinha está em acordo com o preconizado pela legislação vigente.

Deste modo, com os resultados apresentados fica evidente que o método de secagem em camada de espuma é uma alternativa eficiente para produzir e comercializar a guavira e outras frutas de característica sazonal, além de abrir a possibilidade para a produção de novos produtos da farinha de polpa de guavira. Contudo são necessários estudos mais amplos que comprovem a validade do método para outras frutas, contribuindo assim para a expansão da cadeia de suprimentos da indústria alimentícia.

\section{Referências}

ALEXANDRE, H. V. Secagem da polpa de pitanga e armazenamento do pó. 2005. 108 f. Tese de Doutorado. Dissertação (Mestrado em Engenharia Agrícola)-Universidade Federal de Campina Grande, Campina Grande. 2005.

ALVES, A.; DIAS, T.; HASSIMOTTO, N.; NAVES, M. Ascorbic acid and phenolic contents, antioxidant capacity and flavonoids composition of Brazilian Savannah native fruits. Food Science and Technology, v. 37, 564-569, 2017.

ANVISA. BRASIL. Agência Nacional de Vigilância Sanitária. Consulta Pública nº 80. Diário Oficial da União - Brasília, DF, Brasil, 2019.

ARAL, S; BEŞE, A. V. Convective drying of hawthorn fruit (Crataegus spp.): effect of experimental parameters on drying kinetics, color, shrinkage, and rehydration capacity. Food chemistry, v. 210, p. 577-584, 2016.

ARAÚJO, C. S; MACEDO, L. L; VIMERCATI, W. C; OLIVEIRA, A. DO N; E SARAIVA, S. H. Cinética de secagem de acerola (Malpighia emarginata DC) pelo processo foammat. Revista Univap, 22(40), 206. 2016. 
ARAÚJO, W. D; GONELI, A. L. D; CORRÊA, P. C; FILHO, C. P. H; MARTINS, E. A. S. Foam-mat drying kinetics for acerola and adjustment of the mathematical models. Brazilian Journal of Food Technology, v.20, 2017. n.17, 2017.

BACCAR, L. C. M. Equilíbrio do pH e saúde, medicina alternativa. Portal da Educação. 2016.

BAPTESTINI, F. M.; CORREAA, P. C.; JUNQUEIRA, M. S.; RAMOS, A. M; VANEGAS, J. D; B. COSTA, C. F. Modelagem matemática da secagem de espuma de graviola. Revista Brasileira de Engenharia Agrícola e Ambiental, v.19, p.1203-1208, 2015.

BRASIL. Consulta Pública $n^{\circ}$ 80, de 13 de dezembro de 2004. Regulamento técnico sobre ingestão diária recomendada (IDR) de proteína, vitaminas e minerais. Brasília: Agência Nacional de Vigilância Sanitária - ANVISA, 2004.

BREDA, C. A. Desidratação da polpa do fruto da guavira (campomanesia adamantium) pelo processo em camada de espuma. Dissertação (Mestrado em Ciência e Tecnologia Ambiental). Universidade Federal da Grande Dourados. Dourados - MS. 2011.

CARDOSO, I. R. M; ZUNIGA, A. D. G; FRONZA, P; MACIEL, A. G; E FERREIRA, J. DA S. Análise da cinética e modelagem matemática da secagem da polpa de buriti (Mauritia flexuosa). Engevista, v. 19, n. 5, 1188-1197. 2017.

CARDOSO, J. A. D. C; ROSSALES, R. R; LIMONS, B; REIS, S. F; SCHUMACKER, B. D. $\mathrm{O}$; e HELBIG, E. Teor e estabilidade de vitamina $\mathrm{C} e m$ sucos in natura $\mathrm{e}$ industrializados. Mundo saúde (Impr.), v. 39, n.4, 460-469. 2015.

CELESTINO, S.M.C. Princípios de Secagem de Alimentos. Embrapa Cerrados. Planaltina. p $10,2010$.

COSTA, L. M; RESENDE, O; GONÇALVES, D. N; \& DE OLIVEIRA, D. E. C. Modelagem matemática da secagem de frutos de crambe em camada delgada. Bioscience Journal, 31(2). 2015 .

CUNHA, M. F; RIBEIRO, L. M. P; DAMASCENO, K. A; ALVES, A. N; GONÇALVES, R. M. S; E GONÇALVES, C. A. A. Acidez, sua relação com pH e qualidade de geleias e doces em barra. Boletim técnico IFTM, n. 2, p. 14-19. 2016.

DEMENCIANO, S.C; NANTES, E. C. S; CAMPOS, R. P; MILÃO, E,C; CALARGE, A. Parâmetros físico-químicos a polpa de guavira congelada. Anais de congresso. Simpósio Latino Americano de Ciências de Alimentos, v. 2. 2015.

DRESCH, D. M; SCALON, S. P. Q; MUSSURY, R. M; e MASETTO, T. E. Do desiccation and storage of Campomanesia adamantium (Cambess.) O. Berg (Myrtaceae) seeds affect the formation and survival of seedlings. African Journal of Agricultural Research, v. 10 n. 33, 3216-3224. 2015.

ELIAS, N. D. F; BERBERT, P. A; MOLINA, M. A. B. D; VIANA, A. P; DIONELLO, R. G; \& QUEIROZ, V. A. V. Avaliação nutricional e sensorial de caqui cv Fuyu submetido à desidratação osmótica e secagem por convecção. Food Science and Technology, 28(2), 322328. 2008. 
EMBRAPA Florestas. Valor Nutricional da Guabiroba. Conservação da Biodiversidade e Valoração da Floresta de Araucária. Embrapa Florestas. Columbo - PR. 2015.

FERREIRA, C. N. P. Determinação da Análise Química e Propriedades Emulsificantes e Espumantes da Farinha de Girassol. Trabalho de Conclusão (Graduação em Engenharia Química). Instituto municipal de Ensino Superior de Assis - IMESA e Fundação Educacional do Município de Assis - FEMA, Assis - SP, 2011.

FOOD INGREDIENTS BRASIL. Aplicações do ácido cítrico na indústria de alimentos. Revista Food Ingredients Brasil, v. 1, n. 30. 2014.

FRANCO, T. S; PERUSSELLO, C. A; ELLENDERSEN, L. N; \& MASSON, M. L. Effects of foam mat drying on physicochemical and microstructural properties of yacon juice powder. LWT-Food Science and Technology, 66, 503-513, 2016.

FREITAS, B. S.; CAVALCANTE, M. D.; CAGNIN, C.; SILVA, R. M. D; Plácido, G. R; de Oliveira, D. E. Physical-chemical characterization of yellow mombin (Spondias mombin L.) foam-mat drying at different temperatures. Revista Brasileira de Engenharia Agrícola e Ambiental, v. 22, n. 6, p. 430-435, 2018.

GASPARIN, P. P; CHRIST, D; COELHO, S. R. M. Secagem de folhas Mentha piperita em leito fixo utilizando diferentes temperaturas e velocidades de ar. Revista Ciência Agronômica, v. 48, n. 2, p. 242-250, 2017.

GONELLA, J. S. L. Análise dos processos de negócios da gestão de cadeia de suprimentos de polpa de frutas a partir da relação entre agroindústria processadora e produtor rural familiar. Dissertação (Mestrado em Agronegócio e Desenvolvimento). Universidade Estadual Paulista "Júlio de Mesquita Filho". Tupã - SP, 2018.

GUIMARÃES, M. K. A; FIGUEIRÊDO, R; QUEIROZ, MELO, A. J. de. Foam-mat drying kinetics of keitt mango pulp. Revista Caatinga, v. 30, n. 1, p. 172-180, 2017.

HENDERSON, S. M.; PABIS, S. Grain drying theory I. Temperature effect on drying coefficient. Journal of Agricultural Engineering Research, v. 6, n. 3, p. 169-174, 1961.

IAL (INSTITUTO ADOLFO LUTZ). Métodos físico-químicos para análise de alimentos. 4 ed. São Paulo: IAL, 2008. 1018p.

IBGE. Estudo nacional da despesa familiar - ENDEF: Tabela de Composição dos Alimentos. ed. 5. Rio de Janeiro, 1999, p. 49.

LEAL, A. K; GERALDI, C. A. Q; KLASSEN, T. influência da temperatura de secagem através do método de camada de espuma, na concentração de ácido ascórbicos, nas polpas dos frutos de abacaxi, acerola e carambola. XVI Encontro Brasileiro sobre Ensino de Engenharia Química. Fortaleza - CE. 2016.

LOPES, A. C. S.; MENEZES, M. C.; M. ARAÚJO, L. O ambiente alimentar e o acesso a frutas e hortaliças: "Uma metrópole em perspectiva". Saúde e Sociedade. v.26, p 3, 2017.

MACHADO, A. M; DE SOUZA, M. C; DA SILVA, M; JUNQUEIRA, S. H. S; \& TEIXEIRA, L. J. Q. Cinéticas de secagem do abacaxi cv. Pérola. Enciclopédia Biosfera, Centro Científico Conhecer, Goiânia, v.8, n.15; p. 428. 2012. 
MAYER, R. Caracterização físico-química das sementes de araçá-amarelo e potencial antioxidante do óleo das sementes em óleo de girassol induzido à oxidação, Dissertação (Mestrado em Ciência e Tecnologia de Alimentos). Universidade Estadual de Ponta Grossa, Ponta Grossa, 2015.

MIDILLI, A.; KUCUK, H.; YAPAZ, Z. A new model for single-layer drying. Drying Technology, New York, v.20, n.7, p.1503-1513, 2002.

MOSCON, E. S; MARTIN, S; SPEHAR, C. R; DEVILLA, I. A; E JUNIOR, F. RM; Cinética de secagem de grâos de quinoa (Chenopodium quinoa W.). Revista Engenharia na Agricultura-Reveng, v. 25, n. 4, p. 318-325, 2017.

NASCIMENTO, V. R; BIAGI, J. D; OLIVEIRA, R. de. Modelagem matemática da secagem convectiva com radiação infravermelha de grãos de Moringa oleifera. Revista Brasileira Engenharia Agrícola Ambiental, v. 19, p. 686-692, 2015.

NUNES, J. S; LINS, A. D. F; GOMES, J. P; DA SILVA, W. P; \& DA SILVA, F. B. Influência da temperatura de secagem nas propriedades físico-química de resíduos abacaxi. Agropecuária Técnica, v. 38, n. 1, 41-46. 2017.

OLIVEIRA, J. D. D; ALVES, D. K. M; MIRANDA, M. L. D; ALVES, J. M; XAVIER, M. N; CAZAL, C. D. M; e ALVES, C. C. F. Chemical composition of essential oil extracted from leaves of Campomanesia adamantium subjected to different hydrodistillation times. Ciência Rural, v. 47, n. 1. 2017.

OSHIRO, A. M. Conservação Pós-Colheita De Frutos E Sementes De Guavira (Campomanesia adamantium Camb.) Em Diferentes Embalagens e Temperaturas. Tese (Doutorado em Agronomia). Universidade Federal da Grande Dourados, Dourados - MS, 2012.

PAGE, G. E. Factors influencing the maximum of air-drying shelled corn in thin layer. Thesis Dissertation (M.Sc.) - Purdue University, Indiana, 1949.

PEIXOTO, F. N; PINHEIRO, J. A. Gestão de estoques um estudo de caso sobre controle das perdas de produtos hortifrutigranjeiros. Diálogos - Economia e Sociedade. v.1, n 1, 2017.

PEREIRA, B. M.; BRAVIN, G. P. O.; OLIVO, A. M. Aplicação do controle da qualidade no setor de hortifrúti de um supermercado visando a redução de perdas. Colloquium Exactarum, v. 9, n. Especial, Jul-Dez, 2017, p. 172- 177. Presidente Prudente - SP, 2017.

RESENDE, K. K. O; SOUSA, S; GUEDES, S. F; E LOSS, R. A. Cinética de secagem e avaliação físico-química de fruta-pão (Artocarpus altilis) variedade seminífera. Journal Of Neotropical Agriculture, v. 6, n. 1, p. 74-81. 2019.

RIGUETO, C. V. T; EVARISTO, L. M; GERALDI, C. A. Q; \& COVRE, L. Influência da temperatura de secagem de uvaia (Eugenia pyriformis) em camada de espuma. Engevista, v.20, n.4, p. 537-547, 2018.

ROCHA, W. S; LOPES, R. M; SILVA, D. D; VIEIRA, R. F; SILVA, J. D; e AGOSTINICOSTA, T. D. S. Compostos fenólicos totais e taninos condensados em frutas nativas do cerrado. Revista Brasileira de Fruticultura, v. 33, n.4, 1215-1221. 2011. 
SANTOS, R. F. Aproveitamento de frutas nativas para elaboração de farinhas e incorporação em biscoitos tipo cookies. $88 \mathrm{f}$. Dissertação (Mestrado em Tecnologia de Alimentos) - Universidade Tecnológica Federal do Paraná, Londrina, 2018.

SCALON, S. P. Q; OSHIRO, A. M; DRESCH, D. M. Conservação pós-colheita de guavira (Campomanesia adamantium Camb.) Sob diferentes revestimentos e temperaturas de armazenamento. Revista Brasileira de Fruticultura, v. 34, n. 4, p. 1022-1029, 2012.

SEAB - SECRETARIA DA AGRICULTURA E ABASTECIMENTO. Análise da conjuntura agropecuária safra 2016/17. Andrade, P. F. S. Departamento rural, Estado do Paraná. 2017.

SILVA FILHO, E. D. S.; FIGUEIRÊDO, R. M. F.; QUEIROZ, A. J. M.; GUIMARÃES, M. K. A. Cinética de secagem em camada de espuma da polpa da manga cv. Haden. Comunicata. Scientiae; Bom Jesus, v.7, n.3, p.354-361, Ago./Out. 2016.

SILVA, J. A. S. L. Desidratação de ervas condimentares: análise do processo de secagem. Trabalho de Conclusão de Curso Graduação em Nutrição. Universidade Federal de Pernambuco. Vitória de Santo Antão. 2019.

SILVA, M. R.; LACERDA, D. B. C. L.; SANTOS, G. G.; MARTINS, D. M. O. Caracterização química de frutos nativos do cerrado. Ciência Rural, Santa Maria, v. 38, n. 6, p. 1790-1793, 2008.

TELLES, E, O. Microrganismos deteriorantes, patogênicos e tecnológicos - fatores intrínsecos e extrínsecos. VPS 22012 - Higiene e Segurança Alimentar. 2012.

VALLILO, M. I; LAMARDO, L. C. A; GABERLOTTI, M. L; OLIVEIRA, E. D; e MORENO, P. R. H. Composição química dos frutos de Campomanesia adamantium (Cambessédes) $\mathrm{O}$. Berg. Ciência e tecnologia de alimentos, v. 26, n. 4, 725-955. 2006.

VIEIRA, M; PELloSO, I. A; ZÁRATE, N. A. H; DAVIDE, L. M. C; SANTOS, M. C; \& CARNEVALI, T. Diversidade fenotípica em caracteres de frutos e germinação de sementes de Campomanesia adamantium Camb. Revista de la Facultad de Agronomía, 117. 2018.

WIJEWARDANA, R. M. N. A; NAWARATHNE， S. B; WICKRAMASINGHE， I; GUNAWARDANE, C. R; WASALA, W. M. C. B; \& THILAKARATHNE, B. M. K. S. Retention of physicochemical and antioxidant properties of dehydrated bael (Aegle marmelos) and palmyra (Borassus flabellifer) fruit powders. Procedia Food Science, v. 6, 170-175. 2016. 